\title{
Reference-Electrode Free pH Sensing Using Impedance Spectroscopy ${ }^{\dagger}$
}

\author{
Laura Folkertsma 1, Lennart Gehrenkemper ${ }^{1}$, Jan Eijkel 1, Karin Gerritsen ${ }^{2}$ and Mathieu Odijk 1,* \\ 1 BIOS-Lab on a Chip Group, MESA+ Institute of Nanotechnology, Technical Medical Centre, Max Planck \\ Center for Complex Fluid Dynamics, University of Twente, 7500 AE Enschede, The Netherlands; \\ 1.folkertsma@utwente.nl (L.F.); 1.gehrenkemper@uni-muenster.de (L.G.); j.c.t.eijkel@utwente.nl (J.E.) \\ 2 Department of Nephrology and Hypertension, University Medical Center Utrecht, 3508 GA Utrecht, \\ The Netherlands; K.G.F.Gerritsen@umcutrecht.nl \\ * Correspondence: M.Odijk@utwente.nl; Tel.: +31-534-894-620 \\ + Presented at the Eurosensors 2018 Conference, Graz, Austria, 9-12 September 2018.
}

Published: 30 November 2018

\begin{abstract}
We present a reference-electrode free sensor able to measure both $\mathrm{pH}$ and conductivity based on impedance spectroscopy. The electrode is made of a layer of indium-tin-oxide (ITO). The impedance of this electrode at low frequencies depends on its double layer capacity, which varies with $\mathrm{pH}$ due to modification of oxide groups at the ITO surface. At high frequencies, the impedance is determined by the resistance in the system, which corresponds to the inverse conductivity of the solution. Because no reference electrode is needed for this technique, miniaturization of the $\mathrm{pH}$ sensor is simple. We demonstrate a proof-of-principle experiment of the sensor for human plasma $\mathrm{pH}$ measurements.
\end{abstract}

Keywords: pH sensor; impedance spectroscopy; conductivity; indium tin oxide; double layer capacitance; human plasma

\section{Introduction}

Knowledge of the $\mathrm{pH}$ and conductivity of a solution is relevant for many applications and determinations. In dialysis especially, the acid-base status of the patients' blood is monitored to control the treatment quality. Furthermore, in sorbent dialysis as applied in portable and wearable artificial kidneys, there is a need for incorporation of $\mathrm{pH}$ and conductivity sensors to monitor dialysate quality. Since the greatest challenge in developing these artificial kidneys is miniaturization of the device, miniaturization of the sensors is also crucial. While conductivity sensors are generally easy to miniaturize, miniaturization of $\mathrm{pH}$ sensors has been an ongoing project for many years. Standard pH electrodes, as well as ion-sensitive field effect transistors (ISFETS) are based on potentiometric techniques and hence require a reference electrode. Stable reference electrodes generally contain an inner electrolyte, which severely limits the options for miniaturization of reference electrodes for extended use. For ISFETS, attempts have been made at fabricating so called REFETS, reference-electrode field effect transistors, but long-term stability of these remains problematic[1].

We have found that by using impedance spectroscopy as the measurement technique instead of potentiometry, we can circumvent the need for a reference electrode. Using indium-tin-oxide (ITO) layers as the sensing element, we have been able to obtain promising results in the determination of $\mathrm{pH}$ on artificial buffers, as well as on human plasma. By measuring the impedance at only two frequencies, we sense the $\mathrm{pH}$, as well as the conductivity of our samples.

The principle behind the $\mathrm{pH}$ sensing is the change of the ITO surface charge due to (de)protonation of the oxide groups. This is reflected in a change in the double layer. Since impedance 
measurements at the right frequency probe the double layer capacitance, they are sensitive to these changes. We are developing a model to theoretically describe our measured data. In this model, we use the equivalent circuit shown in Figure 1 to represent the ITO electrode and sample. The model is based on previous work of our group [2,3].

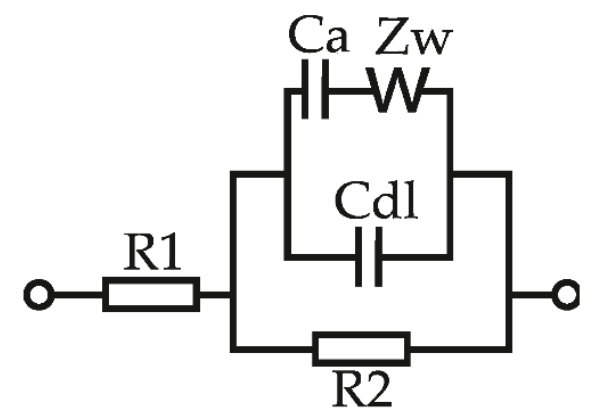

Figure 1. Equivalent circuit used for theoretical modeling of the acquired data. R1 is the electrolyte resistivity, R2 the interfacial resistance, $\mathrm{Cd}$ l the diffuse layer capacitance; $\mathrm{Ca}$ the capacitance due to absorbed charge (protons) and $\mathrm{Zw}$ a Warburg element representing thediffusion of protons towards the surface.

\section{Materials and Methods}

\subsection{Chemicals}

Phosphate buffered saline (PBS) tablets were ordered from Sigma-Aldrich (Zwijndrecht, the Netherlands). Dissolving 1 tablet in $200 \mathrm{~mL}$ DI gives $0.01 \mathrm{M}$ phosphate buffer; $0.0027 \mathrm{M} \mathrm{KCl} ; 0.137 \mathrm{M}$ $\mathrm{NaCl}$; pH 7.4 at $25{ }^{\circ} \mathrm{C}$. PBS buffers at various $\mathrm{pH}$ values were prepared by adjusting the $\mathrm{pH}$ of this standard PBS buffer (original pH 7.4) with $\mathrm{Na}_{2} \mathrm{HPO}_{4}$ or phosphoric acid. $0.1 \mathrm{M}$ solutions of $\mathrm{NaOH}$ and $\mathrm{HCl}$ were used to adjust the $\mathrm{pH}$ of the human plasma sample. $\mathrm{NaOH}, \mathrm{HCl}(37 \%$ solution in water), $\mathrm{Na}_{2} \mathrm{HPO}_{4}$ and phosphoric acid ( $\leq 85 \mathrm{wt} \%$ solution in water) were ordered from Sigma-Aldrich and used as received.

\subsection{Impedance Measurements}

Impedance measurements were performed using a bipotentiostat (SP300, Bio-Logic SAS, Seyssinet-Pariset, France) in a two-electrode configuration. ITO-covered glass slides were ordered from Sigma-Aldrich (Surface resistivity $70 \Omega / \mathrm{sq}$ to $100 \Omega / \mathrm{sq}$ ), and cut to $1 \mathrm{~cm} \times 2 \mathrm{~cm}$. Additionally, three parallel shallow cuts were made in the chips, dividing the ITO into four separate lanes, resulting in four electrodes on one chip. During the measurements, three of these four electrodes were used. To prevent electrolyte leaking from the cell through these cuts, drops of UV-curable glue (NOA 81, Norland, Cranbury, NJ, USA) were positioned on the cuts and the glue was allowed to fill the cuts due to capillary interactions. When the glue in the cuts had entered the sensing area and therefore water tightness was ensured, the glue was cured using an UV light (in-house built, $6 \mathrm{~s}$ pulses; $350 \mathrm{~mW}$ $\mathrm{cm}^{-2}, 365 \mathrm{~nm}$ ). During measurements, the first and third ITO lanes were connected and used together as counter (and pseudo-reference) electrode; the second ITO lane was used as working electrode. Impedance spectra were measured at the open circuit potential with an AC disturbance of $10 \mathrm{mV}$. The electrochemical cell used is shown in Figure 2a; an ITO electrode is shown in Figure 2b. 


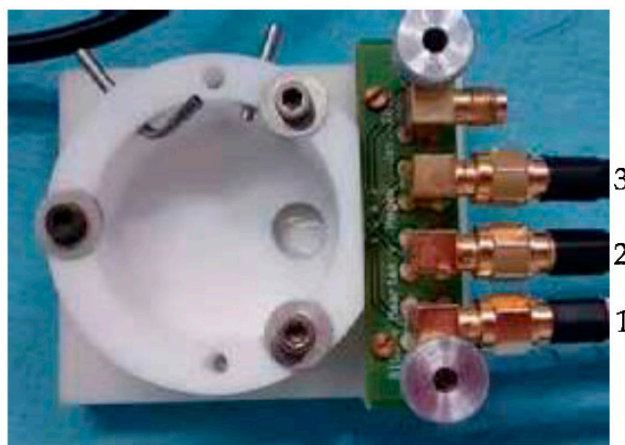

(a)

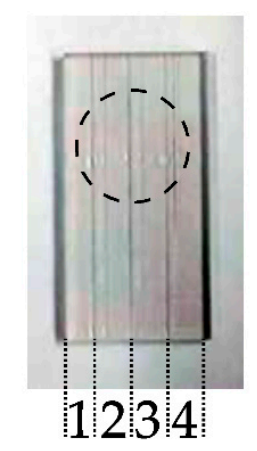

(b)

Figure 2. (a) Electrochemical cell used for impedance measurements on buffers. (b) Electrodes as used in measurements. The dashed circle indicates the area that is exposed to the sample. Lanes 1 and 3 were used as counter electrodes; lane 2 was used as working electrode; lane 4 was not used.

From the full spectrum, the frequencies at which the phase was maximal $\left( \pm 0^{\circ}, 300 \mathrm{kHz}\right)$ and minimal $\left( \pm-80^{\circ}, 10 \mathrm{~Hz}\right)$ were obtained. The impedance at these frequencies was recorded over time while the $\mathrm{pH}$ or concentration of buffer in the cell was varied. $\mathrm{pH}$ sensing experiments were performed in PBS ( $0.01 \mathrm{M}$ phosphate buffer). The starting concentration for the conductivity sensing experiments was the same PBS buffer at $\mathrm{pH}$ 7.3. During the concentration-variations the $\mathrm{pH}$ of this (diluted) buffer varied between 7.3 and 7.5. A commercial pH meter (SevenMulti, Mettler Toledo, Tiel, The Netherlands) was used as a reference to determine the $\mathrm{pH}$ of the solutions in the cell. Because the volume of the solution in the cell was too low to use the $\mathrm{pH}$ meter directly in the cell, identical manipulations as performed on the solution in the cell were performed on a larger volume of the same solution in a separate vial and the $\mathrm{pH}$ of this reference solution was monitored. No reference measurements were performed for the conductivity; only relative concentration and impedance are compared in this work.

Human plasma was obtained from a volunteer after informed consent was provided. The $\mathrm{pH}$ of the sample was adjusted using $0.1 \mathrm{M}$ solutions of $\mathrm{HCl}$ and $\mathrm{NaOH}$. Two measurements at comparable reference $\mathrm{pH}$ values were used to correct for linear drift during theexperiment.

\section{Results and Discussion}

Figure 3 shows the impedances measured at $10 \mathrm{~Hz}$ and $300 \mathrm{kHz}$ while varying either the $\mathrm{pH}$ or the concentration of a phosphate buffered saline solution. This figure reveals the correlation between the $\mathrm{pH}$ or conductivity and the impedance at $10 \mathrm{~Hz}$ and $300 \mathrm{kHz}$, respectively. In Figure 3c, d, the mean plateau value for each plateau is plotted against the corresponding $\mathrm{pH}$ or relative concentration and the least squares linear fits are shown. $\mathrm{R}^{2}$ values of 0.994 and 0.973 indicate the strong linear dependence of impedance on $\mathrm{pH}$ and dilution factor. Figure 4 shows the results of impedance measurements on a human plasma sample in which the $\mathrm{pH}$ was varied by additions of $0.1 \mathrm{M}$ hydrochloric acid and sodium hydroxide. These data show the applicability of the method for clinically relevant samples. We will further improve the sensor by using temperature control and a closed (flow) cell to avoid escape of $\mathrm{CO}_{2}$. 


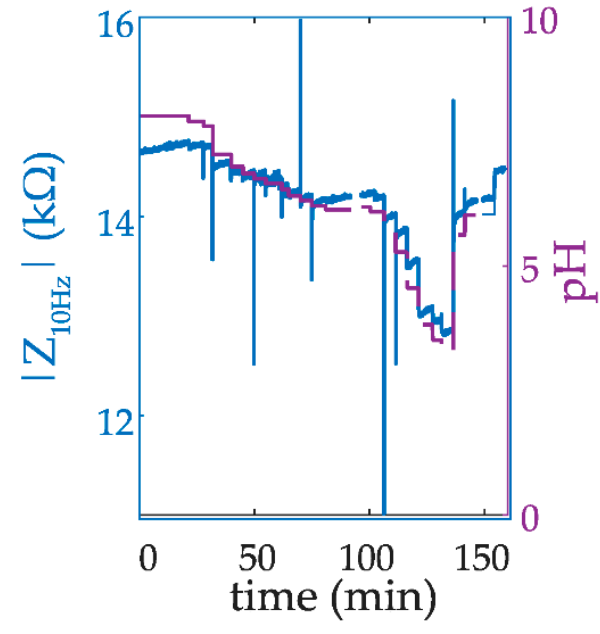

(a)

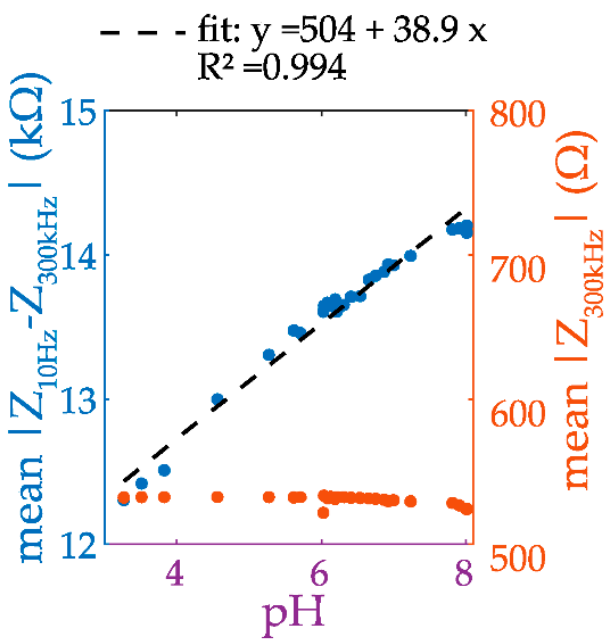

(c)

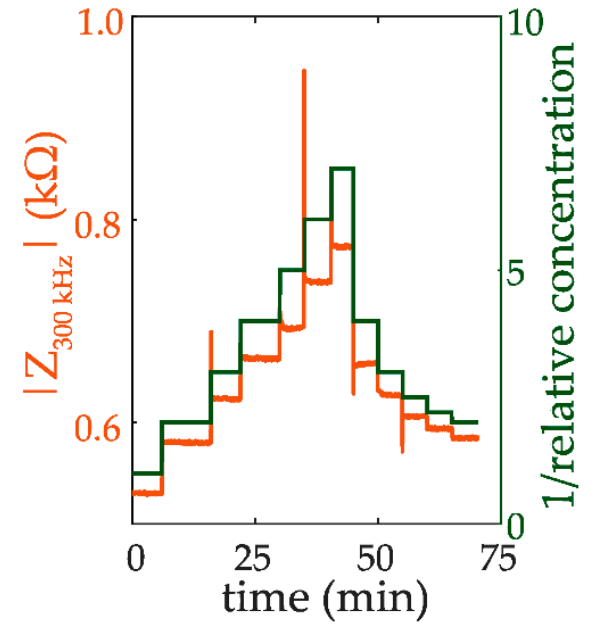

(b)

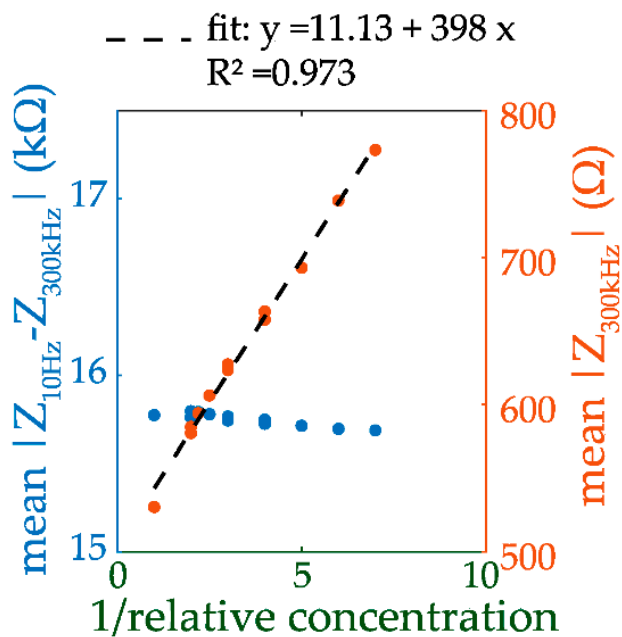

(d)

Figure 3. (a) Impedance of the ITO electrode at $10 \mathrm{~Hz}$ (blue) and $\mathrm{pH}$ (green) vs time. (b) Impedance of the bare ITO electrode at $300 \mathrm{kHz}$ (red) and 1/relative concentration (=dilution factor, green) vs time. (c) Mean IZI values per applied pH value (blue: $10 \mathrm{~Hz}-300 \mathrm{kHz}$; red: $300 \mathrm{kHz}$ ). (d) Mean IZI values per applied dilution factor (blue: $10 \mathrm{~Hz}-300 \mathrm{kHz}$; red: $300 \mathrm{kHz}$ ).

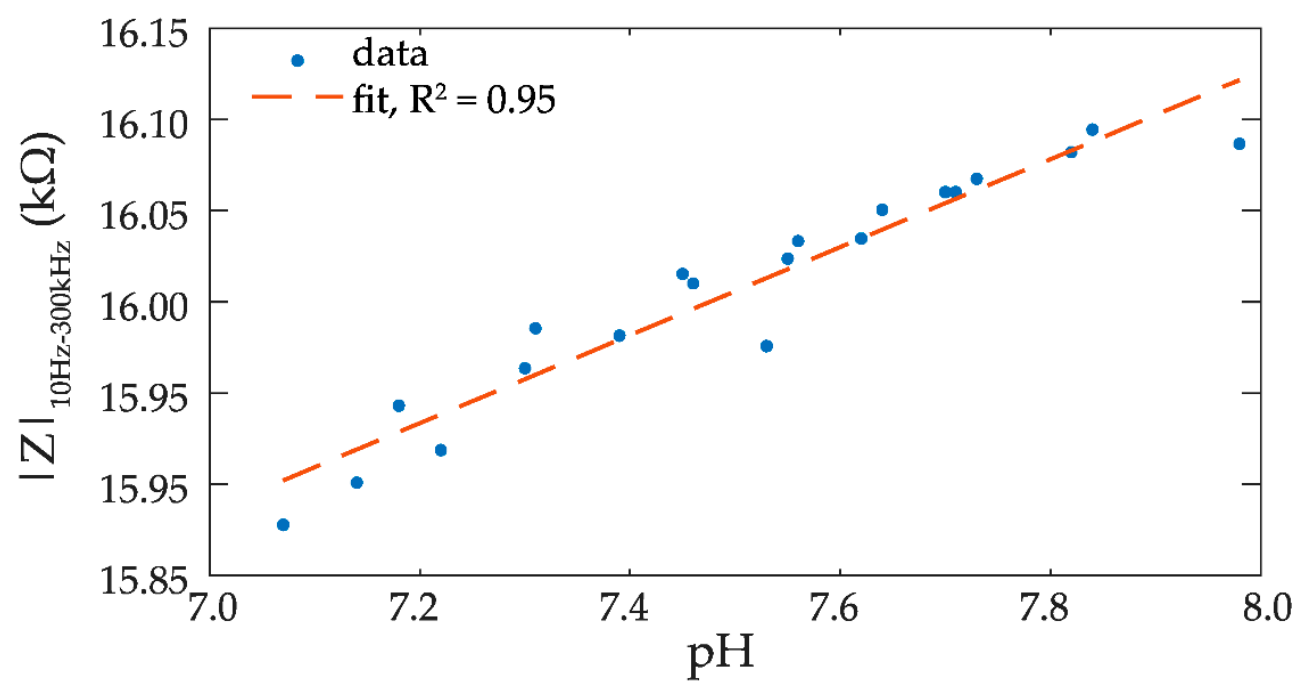

Figure 4. Impedance of the ITO electrode vs $\mathrm{pH}$ of a human plasma sample after removal of drift (3 $\Omega / \mathrm{min}$ ). The $\mathrm{pH}$ of the plasma sample was varied by additions of aqueous sodium hydroxide and hydrochloric acid (both $0.1 \mathrm{M}$ ). Linear least squares fit: $|\mathrm{Z}|=241 \Omega / \mathrm{pH} \cdot \mathrm{pH}+1.42 \mathrm{k} \Omega$. 


\section{Conclusions}

Impedance measurements on ITO electrodes are a promising technique to simultaneously measure $\mathrm{pH}$ and conductivity, without need for a reference electrode. This means that the combined $\mathrm{pH}$ and conductivity sensor will be easy to miniaturize. The resulting miniaturized sensor could be a solution for $\mathrm{pH}$ and conductivity sensing in flow-through applications, such as the portable artificial kidney.

Acknowledgments: This work was funded by project number 728.011.205 from the research programme "NWO ChemThem Out-of-Equilibrium Self-Assembly" and by the financial support of the strategic alliance of the Technical Medical Centre (formerly MIRA institute) of the University of Twente, the University of Utrecht and the University Medical Center Utrecht. LG received a scholarship from the Erasmus Programme.

Conflicts of Interest: The authors declare no conflict of interest. The founding sponsors had no role in the design of the study; in the collection, analyses, or interpretation of data; in the writing of the manuscript, and in the decision to publish the results.

\section{References}

1. Guth, U.; Gerlach F.; Decker, M.; Oelßner, W.; Vonau, W. Solid-state reference electrodes for potentiometric sensors. J. Solid State Electrochem. 2009, 13, 27-39, doi:10.1007/s10008-008-0574-7.

2. Bousse, L.; Bergveld, P. On the impedance of the silicon dioxide/electrolyte interface. J. Electroanal. Chem. 1983, 152, 22-39, doi:10.1016/S0022-0728(83)80030-8.

3. Van Hal, R.; Eijkel, J.; Bergveld, P. A general model to describe the electrostatic potential at electrolyte oxide interfaces. Adv. Colloid Interface Sci. 1996, 69, 31-62. 